\title{
EDITORIAL
}

\section{Recurrent laryngeal nerve injury during patent ductus ligation: can this common complication be reduced?}

\section{Journal of Perinatology (2010) 30, 371-372; doi:10.1038/jp.2010.51}

In this issue of the Journal of Perinatology, Jennifer Benjamin ${ }^{1}$ and colleagues report long-term morbidities associated with injury to the left recurrent laryngeal nerve (RLN) during surgical closure of a patent ductus arteriosus (PDA) in a group of infants whose birth weight was less than $1 \mathrm{~kg}$. This is the first study to do so. The most remarkable aspect of this paper was the unexpectedly high incidence (40\%) of symptomatic vocal cord paralysis. Laryngoscopy in these patients demonstrated left-sided vocal cord paralysis, an observation establishing RLN injury following PDA ligation. When comparing the infants having symptomatic vocal cord paralysis with the asymptomatic PDA-ligated infants, Benjamin et al. demonstrated a significantly increased incidence of long-term morbidities. These morbidities included bronchopulmonary dysplasia (BPD), reactive airway disease, and the need for a gastrostomy tube for persistent ineffective swallowing and aspiration. Several aspects of the study warrant discussion.

First, regarding BPD and its associated reactive airways, the authors suggest that chronic aspiration is an important contributor, as it would prolong the duration of positive pressure ventilation and oxygen therapy. Multiple possible causative mechanisms for aspiration are invoked by Benjamin et al., ${ }^{1}$ but their data show a clear increase in pulmonary morbidity in those patients with post-PDA ligation vocal cord paralysis.

Second, reports of RLN injury vary a good deal from institution to institution. In the present study, RLN injury occurred in $40 \%$ of cases. In their discussion, Benjamin et al. indicate that the reported incidence of vocal cord paralysis ranges from 1.7 to $67 \%$. The variability is likely due, in part, to the fact that nerve injury depends on the gestational age of the infant. Very low birth weight infants are much more susceptable than more mature infants. Also, the relative experience of the surgeon must be a factor as well. Another possibility may be the closure technique. Two closure methods are in use - silk ligature or a clip technique using a metal band. Comparison of PDA closure by ligation with that using a clip, as used in the Benjamin study, has been done in only one report. Mandhan $e t a l^{2}$ identified one patient with vocal cord paralysis in two groups of approximately 34 patients each ( $\sim 3 \%$ incidence) from two institutions, one of whom had used the ligature technique and the other had used the clip technique. This report also quoted an incidence of approximately 4 to $5 \%$ for vocal cord paralysis in two other reports that used either ligature or clip techniques in approximately 20 patients each. Thus, the closure technique is not a likely candidate to explain the wide variation of vocal cord paralysis rates. One likely explanation is the age and birth weight of the infants in these reports. Ductal ligation is technically more difficult in small preterm infants. Another probable explanation for the institutionto-institution variability may be the differing degrees of compulsivity in recognizing the symptoms of RLN injury and its confirmation by direct laryngoscopy, demonstrating left vocal cord paralysis.

Third, why is swallowing dysfunction associated with RLN injury? At first glance this would seem unlikely, as pharyngeal constricting muscles are innervated by the glossopharyngeal nerve. However, during a swallow, the entrance to the larynx is protected from aspiration by constriction of the aryepiglottic muscle, which is innervated by the RLN. This muscle causes closure of the inner arytenoid space and associated structures, thus preventing aspiration. However, sensory innervation of the inner arytenoid space is supplied by the internal laryngeal nerves (which are unrelated to the RLN). Stimulation of the submucosal nerve endings in this space produces airway protective responses (apnea, swallowing, cough). ${ }^{3}$ Apnea or cough during attempted swallowing would disrupt swallowing, and thus interfere with sucking and swallowing needed for efficient nursing.

Fourth, can newer techniques for ductal closure produce better results? Video-assisted surgery has been used to close PDA in preterm infants. This technique involves three small incisions (ports). Infants varied between 23 to 31 weeks gestation, and were all less than $1.5 \mathrm{~kg}$ birth weight. The reported incidence of recurrent laryngeal injury was very low (5\%) in a total of 100 cases. ${ }^{4}$ In this study only symptomatic infants (suspected aspiration, difficulty with swallowing, hoarseness) were laryngoscoped. Since recognition of symptoms is largely subjective it may well be that the infants whose vocal cords were not inspected had nerve injury resulting in longterm morbidity. Nevertheless, this surgical technique offers promise and further study is warrented.

In summary, it is clear that RLN injury during PDA closure is very common and causes significant long-term morbidity that is not previously appreciated. Clinicians should be aware of the subtle signs of RLN injury causing unilateral vocal cord paralysis. Hopefully, awareness of this complication will result in new efforts to reduce its incidence. 


\section{Conflict of interest}

The author declares no conflict of interest.

BT Thach

Department of Pediatrics, Washington University School of Medicine, St Louis, MO, USA

E-mail: thach@kids.wustl.edu

\section{References}

1 Benjamin JR, Smith PB, Cotten CM, Jaggers J, Goldstein RF, Malcolm WF Long-term morbidities associated with vocal cord paralysis after surgical closure of a patent ductus arteriosus in extremely low birth weight infants. J Pediatr 2010; 30: 408-413.

2 Mandhan PL, Samarakkody U, Brown S, Kukkady A, Maoate K, Blakelock R et al. Comparison of suture ligation and clip application for the treatment of patent ductus arteriosus in preterm neonates. J Thorac Cardiovasc Surg 2006; 132 : 672-674.

3 Thach BT. Maturation and transformation of reflexes that protect the laryngeal airway from liquid aspiration from fetal to adult life. Am J Med 2001; 111 $69 \mathrm{~S}-77 \mathrm{~S}$.

4 Hines MH, Raines KH, Payne RM, Covitz W, Cnota JF, Smith TE et al. Video-assisted ductal ligation in premature infants. Ann Thorac Surg 2003; 76: $1417-1420$ 